\title{
Preserving Structural Integrity of the Alar Cartilage in Aesthetic Rhinoplasty Using a Cephalic Turn-in Flap
}

\author{
Craig S. Murakami, MD; Jose E. Barrera, MD; Sam P. Most, MD
}

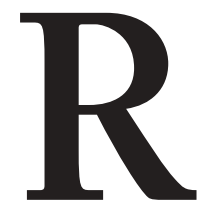

esection of the alar cartilage has long been a mainstay of aesthetic rhinoplasty. One drawback of this technique is the destabilization of the ala/lateral nasal wall complex. Herein we describe the cephalic turn-in flap, a technique for reinforcement of the alar cartilage after removal of its cephalic portion.

Arch Facial Plast Surg. 2009;11(2):126-128

The alar cartilage determines the unique aesthetic norms of the nasal tip. The lower lateral cartilage determines the shape, size, and position of the nasal tip and its relation to the ala and supports the lower lateral nasal wall. One of the most commonly used procedures to reduce volume of the nasal tip is resection of the cephalic portion of the lateral crus of the greater alar cartilage. Overresection of the lateral crus can lead to loss of lateral wall support, loss of tip support, overrotation, alar retraction, and bossae. ${ }^{1,2}$ The structure and support of the lower cartilage may not seem compromised initially; however, longterm stability as a result of contractile forces may render weak cartilages at risk.

An alternative to cephalic resection of the alar cartilage is a turn-in flap of the cephalic portion of the lateral crus. This enables aesthetic corrections and reinforces the durability of the lateral crus, reduces tip volume, and permits medialization of the tip-defining points, thereby achieving a more pleasant-appearing nasal tip. A similar technique has been described specifically for correction of the excessively concave ala and collapse. ${ }^{3}$ Herein, we describe the turn-in flap as an alternative to routine cephalic resection of the lateral crus in aesthetic rhinoplasty and evaluate the advantages and disadvantages of the technique.

Author Affiliations: Division of Otolaryngology-Head and Neck Surgery, Virginia Mason Medical Center, Seattle, Washington (Dr Murakami); and Division of Facial Plastic and Reconstructive Surgery, Stanford University School of Medicine, Stanford, California (Drs Barrera and Most).

\section{METHODS}

The turn-in flap was used in 18 patients for refinement of the nasal tip in cases when loss of structural support of the lateral crus was a particular concern. In 1 patient with functional external valve collapse, the procedure was also used in combination with the bone-anchored suture technique for correction of nasal valve stenosis. ${ }^{4}$

The turn-in flap procedure was performed during open rhinoplasty in all patients. In all cases, the lateral crus was measured and at least $8 \mathrm{~mm}$ of cartilage was preserved. The nasal mucosa was routinely dissected from the inner surface of the lateral crus to reduce the likelihood of folding over of the vestibular skin (Figure 1). The lateral crus was stabilized with 5-0 nonabsorbable mattress sutures. Three mattress sutures were sufficient to fix the folded cephalic portion. Intranasal examination confirmed that the sutures were not exposed intranasally.

\section{RESULTS}

The mean duration of patient follow-up in the series was 9 months. Those who received a turn-in flap exhibited satisfactory nasal tip refinement (Figure 2). The turn-in flap enabled symmetric reduction of the lower lateral cartilages. Additional bulk in the lateral sidewall was not evident, and narrowing of the nasal vestibule was not a problem. There was no associated infection, suture extrusion or visualization, or alar collapse as a result of this procedure. 

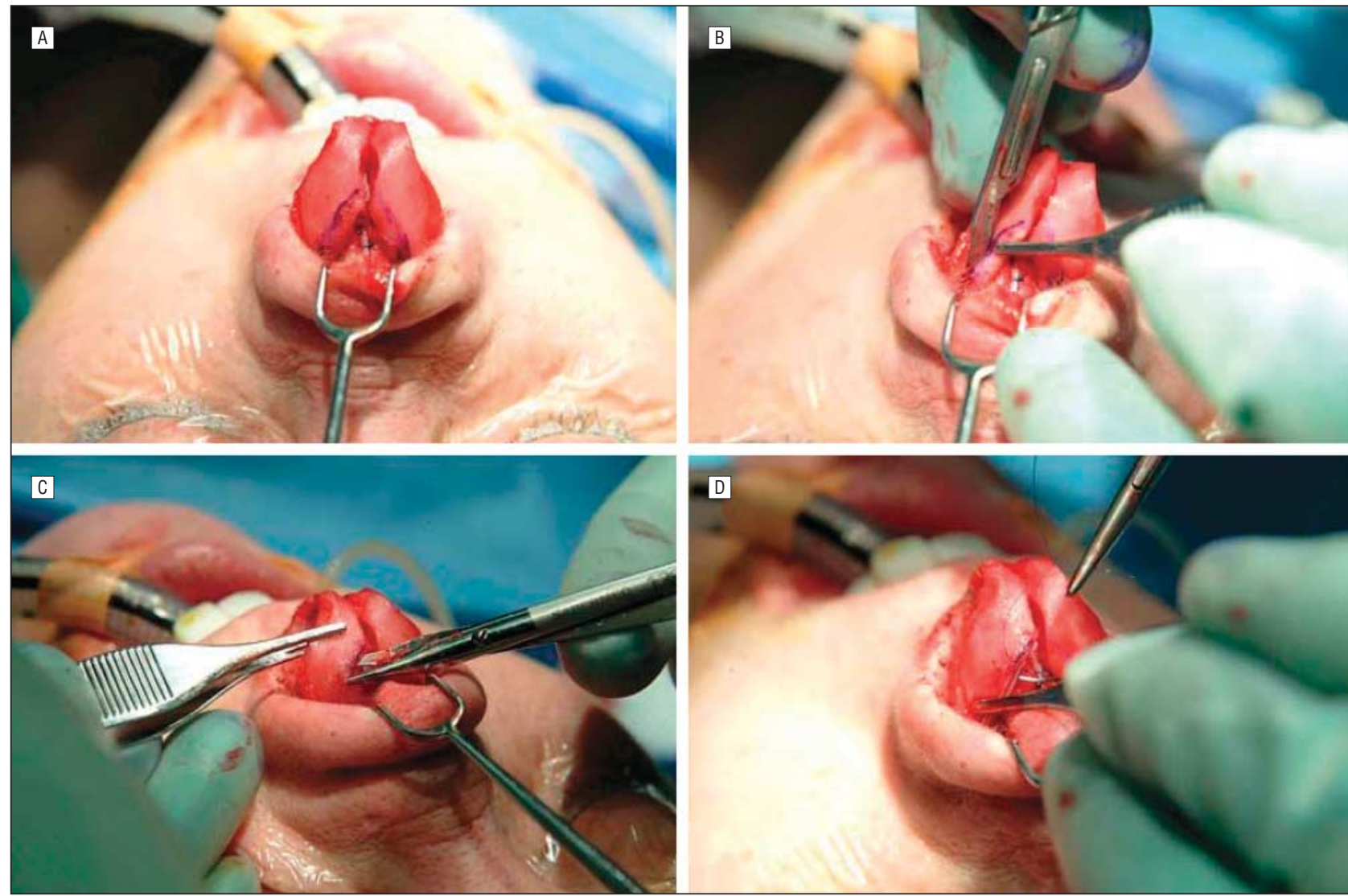

Figure 1. Turn-in flap procedure in refinement of the nasal tip. A, The cephalic portion of the lateral crus is demarcated preserving $8 \mathrm{~mm}$ of cartilage. B, Cartilage is scored with a No. 15 blade scalpel. C, The vestibular skin is dissected free. D, The cartilage is turned in and secured with horizontal mattress sutures.
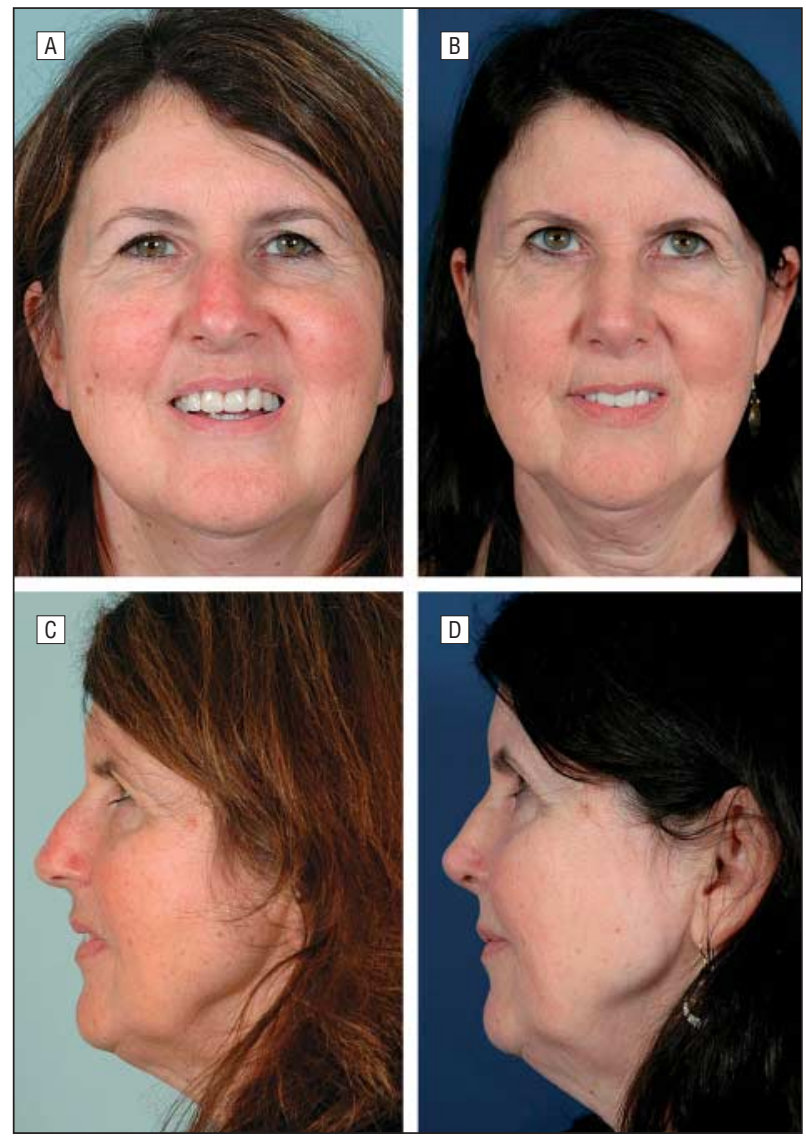

Figure 2. Tip refinement using the cephalic turn-in procedure. A, Anteroposterior view before surgery and 8 months postoperatively (B). C, Left lateral view before surgery and 8 months postoperatively (D).

(REPRINTED) $\overline{\text { ARCH FACIAL PLAST SURG/VOL } 11 \text { (NO. 2), MAR/APR } 2009 \text { WWW.ARCHFACIAL.COM }}$ 


\section{COMMENT}

Nasal tip refinement procedures may lead to weakness of the lateral crus through overresection of the cephalic portion of the lateral crus. This can lead to formation of bossae, alar retraction, and alar collapse. ${ }^{2,5}$ Excessive resection of the alar cartilage and failure to eliminate interdomal width may have a role in the development of bossae, which result after contracture of an overly narrowed complete rim strip. Weakening of the lateral cartilage can result in lateral collapse with negative pressure forces created during inspiration. An anatomical study of the alar base showed that a thin alar rim is present in $20 \%$ of the general patient population. ${ }^{6}$

The turn-in flap used in cephalic narrowing of the lateral crus can improve nasal tip and nasal base support and strength. This procedure can be used in individuals with weak cartilage and a wide lateral crus, giving the surgeon a margin of safety to prevent the aforementioned complications. One exception to this is vertical, superior retraction of the ala, caused by the skeletal void between the upper lateral cartilage and the alar cartilage. The procedure can also be used with functional correction of the internal and external valve by incorporating spreader grafts or the bone-anchored suture technique. The turn-in flap is nondestructive, reversible, and incremental. Additional advantages of the technique are the correction of excessively convex or concave cartilage with horizontal mattress sutures and there is no need for additional graft material. This procedure cannot be used in individuals who have already undergone overresection of the lateral crus because not enough cartilage may be left for turn-in. We have found the turn-in flap to be an improvement over classic cephalic resection of the lateral crus in tip refinement. Long-term and larger outcome studies are required, how- ever, to more definitely evaluate the turn-in flap procedure and to compare it with other techniques.

Accepted for Publication: September 9, 2008.

Correspondence: Sam P. Most, MD, Division of Facial Plastic and Reconstructive Surgery, Stanford University School of Medicine, 801 Welch Rd, Stanford, CA 94305 (smost@ohns.stanford.edu).

Author Contributions: Dr Murakami had full access to all of the data in the study and takes responsibility for the integrity of the data and the accuracy of the data analysis. Study concept and design: Murakami, Barrera, and Most. Acquisition of data: Murakami, Barrera, and Most. Analysis and interpretation of data: Murakami. Drafting of the manuscript: Murakami, Barrera, and Most. Critical revision of the manuscript for important intellectual content: $\mathrm{Mu}-$ rakami and Most. Administrative, technical, and material support: Murakami and Barrera. Study supervision: Most. Financial Disclosure: None reported.

\section{REFERENCES}

1. Gillman GS, Simons RL, Lee DJ. Nasal tip bossae in rhinoplasty: etiology, predisposing factors, and management techniques [published correction appears in Arch Facial Plast Surg. 1999;1(3):170]. Arch Facial Plast Surg. 1999;1(2):83-89.

2. Thomas JR, Tardy ME Jr. Complications of rhinoplasty. Ear Nose Throat J. 1986; 65(1):19-34.

3. Tellioglu AT, Cimen K. Turn-in folding of the cephalic portion of the lateral crus to support the alar rim in rhinoplasty. Aesthetic Plast Surg. 2007;31(3):306-310.

4. Roofe SB, Most SP. Placement of a lateral nasal suspension suture via an external rhinoplasty approach. Arch Facial Plast Surg. 2007;9(3):214-216.

5. Simons RL, Gallo JF. Rhinoplasty complications. Facial Plast Surg Clin North Am. 1994;2(4):521-529.

6. Becker D. Complications of rhinoplasty. In: Papel I, ed. Facial Plastic and Reconstructive Surgery. New York, NY: Thieme Medical Publishers, Inc; 2002: 452-460

Visit www.archfacial.com. As an individual subscriber you can perform Searches. You can perform a simple search using the Search field that appears at the top of every page. This field will search the journal only, unless you search from the JAMA \& Archives Journals level pages, in which case they will search across all 11 journals. Advanced Search enables you to search by citation, title, author, keywords, and date ranges. You can search by journal selection or by topic collection. Finally, you can choose to search only tables and figures. 